\title{
Comparison of regularization methods for solving the Cauchy problem associated with the Helmholtz equation
}

\author{
L. Marin ${ }^{1, * \dagger}$, L. Elliott ${ }^{2}$, P. J. Heggs ${ }^{3}$, D. B. Ingham ${ }^{2}$, D. Lesnic ${ }^{2}$ and X. Wen ${ }^{1}$ \\ ${ }^{1}$ School of the Environment, University of Leeds, Leeds LS2 9JT, U.K. \\ ${ }^{2}$ Department of Applied Mathematics, University of Leeds, Leeds LS2 9JT, U.K. \\ ${ }^{3}$ Department of Chemical Engineering, UMIST, P.O. Box 88, Manchester M60 1QD, U.K.
}

\begin{abstract}
SUMMARY
In this paper, several boundary element regularization methods, such as iterative, conjugate gradient, Tikhonov regularization and singular value decomposition methods, for solving the Cauchy problem associated to the Helmholtz equation are developed and compared. Regularizing stopping criteria are developed and the convergence, as well as the stability, of the numerical methods proposed are analysed. The Cauchy problem for the Helmholtz equation can be regularized by various methods, such as the general regularization methods presented in this paper, but more accurate results are obtained by classical methods, such as the singular value decomposition and the Tikhonov regularization methods. Copyright (C) 2004 John Wiley \& Sons, Ltd.
\end{abstract}

KEY WORDS: boundary element method; Cauchy problem; Helmholtz equation; regularization; inverse problem

\section{INTRODUCTION}

The Helmholtz equation arises naturally in many physical applications related to wave propagation and vibration phenomena. It is often used to describe the vibration of a structure [1], the acoustic cavity problem [2], the radiated wave [3] and the scattering of a wave [4]. Another important application of the Helmholtz equation is the problem of heat conduction in fins, see e.g. References [5-7], and we focus on the later problem in this study.

The well-posedness of the direct problems of the Helmholtz equation via the removal of the eigenvalues of the Laplacian operator is well established, see e.g. Reference [8]. However, many engineering problems do not belong to this category. In particular, the boundary conditions are often incomplete, either in the form of underspecified and overspecified boundary conditions on different parts of the boundary or the solution is prescribed at some internal

\footnotetext{
*Correspondence to: L. Marin, School of the Environment, University of Leeds, Leeds LS2 9JT, U.K.

${ }^{\dagger}$ E-mail: liviu@env.leeds.ac.uk

Contract/grant sponsor: EPSRC
}

Published online 24 May 2004

Copyright (c) 2004 John Wiley \& Sons, Ltd.

Received 5 February 2003

Revised 15 August 2003

Accepted 15 September 2003 
points in the domain. These are inverse problems, and it is well known that they are generally ill-posed, i.e. the existence, uniqueness and stability of their solutions are not always guaranteed.

Unlike in direct problems, the uniqueness of the Cauchy problem is guaranteed without the necessity of removing the eigenvalues for the Laplacian. However, the Cauchy problem suffers from the non-existence and instability of the solution. A boundary element method (BEM)based acoustic holography technique using the singular value decomposition (SVD) for the reconstruction of sound fields generated by irregularly shaped sources has been developed by Bai [9]. The vibrational velocity, sound pressure and acoustic power on the vibrating boundary comprising an enclosed space have been reconstructed by Kim and Ih [10] who have used the SVD in order to obtain the inverse solution in the least-squares sense and to express the acoustic modal expansion between the measurement and source field. Wang and Wu [11] have developed a method employing the spherical wave expansion theory and a least-squares minimization to reconstruct the acoustic pressure field from a vibrating object and their method has been extended to the reconstruction of acoustic pressure fields inside the cavity of a vibrating object by $\mathrm{Wu}$ and $\mathrm{Yu}$ [12]. DeLillo et al. [13] have detected the source of acoustical noise inside the cabin of a medium-sized aircraft from measurements of the acoustical pressure field inside the cabin by solving a linear Fredholm integral equation of the first kind. Recently, Marin et al. $[14,15]$ have solved the Cauchy problem associated to the Helmholtz equation using the BEM in conjunction with an alternating iterative procedure consisting of obtaining successive solutions to well-posed mixed boundary value problems and with the conjugate gradient method (CGM), respectively.

The purpose of this paper is to describe and compare several boundary element regularization methods, such as iterative, conjugate gradient, Tikhonov regularization and singular value decomposition methods, for solving the Cauchy problem for the Helmholtz equation. The regularization is obtained by matching the number of iterations performed, the choice of the regularization parameter, or the choice of the optimal truncation number to the level of the noise in the input data. Regularizing stopping criteria are developed by using the residual of the ill-conditioned system of linear equations obtained from the BEM discretization, in conjunction with the discrepancy principle.

\section{MATHEMATICAL FORMULATION}

Referring to heat transfer for the sake of the physical explanation, we assume that the temperature field $T(\underline{x})$ satisfies the Helmholtz equation in an open bounded domain $\Omega \subset \mathbb{R}^{d}$, where $d$ is the dimension of the space in which the problem is posed, namely

$$
L T(\underline{x}) \equiv\left(\Delta+k^{2}\right) T(\underline{x})=0, \quad \underline{x} \in \Omega
$$

where $k=\alpha+\mathrm{i} \beta \in \mathbb{C}, \mathrm{i}=\sqrt{-1}$.

The general equation describing the temperature distribution along an isolated fin for steadystate two-dimensional heat transfer is obtained by completing a heat balance over an incremental surface element, $\mathrm{d} y=\left(y_{1}-\mathrm{d} y_{1} / 2, y_{1}+\mathrm{d} y_{1} / 2\right) \times\left(y_{2}-\mathrm{d} y_{2} / 2, y_{2}+\mathrm{d} y_{2} / 2\right)$, at some point, $\underline{y}=\left(y_{1}, y_{2}\right)$ within the fin surface, see e.g. References [5-7]. The heat balance over the 
increment, $\mathrm{d} \underline{y}$, for steady-state heat flow is given by

$$
\begin{aligned}
& \left(\dot{Q}_{\mathrm{f}}\left(y_{1}-\mathrm{d} y_{1} / 2, y_{2}\right)-\dot{Q}_{\mathrm{f}}\left(y_{1}+\mathrm{d} y_{1}, y_{2} / 2\right)\right) \mathrm{d} y_{2} \\
& \quad+\left(\dot{Q}_{\mathrm{f}}\left(y_{1}, y_{2}-\mathrm{d} y_{2} / 2\right)-\dot{Q}_{\mathrm{f}}\left(y_{1}, y_{2}+\mathrm{d} y_{2} / 2\right)\right) \mathrm{d} y_{1}-\dot{Q}_{c}\left(y_{1}, y_{2}\right)=0
\end{aligned}
$$

where $\dot{Q}_{\mathrm{f}}\left(y_{1}, y_{2}\right)$ is the conductive heat flow at $y=\left(y_{1}, y_{2}\right)$ and $\dot{Q}_{c}\left(y_{1}, y_{2}\right)$ is the heat lost from the incremental surface of the fin. From the Postulation of Fourier, we have

$$
\dot{Q}_{\mathrm{f}}\left(y_{1}, y_{2}\right)=-\lambda_{\mathrm{f}} A_{\mathrm{f}} \nabla \theta_{\mathrm{f}}\left(y_{1}, y_{2}\right)
$$

where $\lambda_{\mathrm{f}}$ is the thermal conductivity of the fin and $A_{\mathrm{f}}$ is the cross-sectional area of the fin, and by using a Taylor-series expansion around $y=\left(y_{1}, y_{2}\right)$ and neglecting terms after the first-order derivatives we obtain

$$
\begin{aligned}
& \left(\dot{Q}_{\mathrm{f}}\left(y_{1}-\mathrm{d} y_{1} / 2, y_{2}\right)-\dot{Q}_{\mathrm{f}}\left(y_{1}+\mathrm{d} y_{1}, y_{2} / 2\right)\right) \mathrm{d} y_{2} \\
& \quad+\left(\dot{Q}_{\mathrm{f}}\left(y_{1}, y_{2}-\mathrm{d} y_{2} / 2\right)-\dot{Q}_{\mathrm{f}}\left(y_{1}, y_{2}+\mathrm{d} y_{2} / 2\right)\right) \mathrm{d} y_{1}=\nabla \cdot\left(\lambda_{\mathrm{f}} A_{\mathrm{f}} \nabla \theta_{\mathrm{f}}\left(y_{1}, y_{2}\right)\right) \mathrm{d} y_{1} \mathrm{~d} y_{2}
\end{aligned}
$$

The heat lost from the surface of the incremental fin is assumed to be by convection and, by Newton's law of cooling, this is given by

$$
\dot{Q}_{c}\left(y_{1}, y_{2}\right)=h A_{\mathrm{f}}^{\prime}\left(\theta_{\mathrm{f}}\left(y_{1}, y_{2}\right)-\theta_{\infty}\right) \mathrm{d} y_{1} \mathrm{~d} y_{2}
$$

where $h$ is the surface heat transfer coefficient, $A_{\mathrm{f}}^{\prime}$ is the surface area of the fin per unit length and $\theta_{\infty}$ is the temperature of the surrounding medium.

Substitution of Equations (4) and (5) into the heat balance equation (2) provides the following second-order partial differential equation:

$$
\nabla \cdot\left(\lambda_{\mathrm{f}} A_{\mathrm{f}} \nabla \theta_{\mathrm{f}}(\underline{y})\right)-h A_{\mathrm{f}}^{\prime}\left(\theta_{\mathrm{f}}(\underline{y})-\theta_{\infty}\right)=0
$$

The following assumptions are invoked in order to obtain the general fin equation:

(i) The thermal conductivity of the fin, $\lambda_{\mathrm{f}}$, is invariant;

(ii) the surface heat transfer coefficient, $h$, is uniform along the fin;

(iii) the temperature of the surrounding medium, $\theta_{\infty}$, is constant.

Additionally, if we assume that the cross-sectional area of the fin is constant (i.e. the fin thickness, $2 \delta_{\mathrm{f}}$, is invariant) then the fin equation (6) recasts as

$$
\Delta \theta_{\mathrm{f}}(\underline{y})-\frac{h}{\lambda_{\mathrm{f}} \delta_{\mathrm{f}}}\left(\theta_{\mathrm{f}}(\underline{y})-\theta_{\infty}\right)=0
$$

On introducing the following dimensionless variables:

$$
x_{j}=y_{j} / \delta_{\mathrm{f}}, \quad j=1,2, \quad T(\underline{x})=\left(\theta_{\mathrm{f}}(\underline{y})-\theta_{\infty}\right) /\left(\theta_{b}-\theta_{\infty}\right)
$$

where $\theta_{b}$ is the fin base temperature $\left(\theta_{b} \neq \theta_{\infty}\right)$, then the non-dimensional temperature distribution along an isolated fin of constant cross-sectional area for steady-state two-dimensional heat transfer is described by the Helmholtz equation (1) with $k=\alpha+\mathrm{i} \beta, \alpha=0$ and $\beta=\sqrt{h /\left(\lambda_{\mathrm{f}} \delta_{\mathrm{f}}\right)}$. 
We now let $v(\underline{x})$ be the outward normal vector at the boundary $\Gamma=\partial \Omega$ and $\Phi(\underline{x}) \equiv$ $(\partial T / \partial v)(\underline{x})$ be the flux at a point $\underline{x} \in \Gamma$. The Cauchy problem under investigation requires solving the partial differential equation (1) subject to the boundary conditions

$$
T(\underline{x})=\tilde{T}(\underline{x}), \quad \Phi(\underline{x})=\tilde{\Phi}(\underline{x}), \quad \underline{x} \in \Gamma_{2}
$$

where $\tilde{T}$ and $\tilde{\Phi}$ are prescribed functions and $\Gamma_{2} \subset \Gamma$, meas $\left(\Gamma_{2}\right)>0$. In the above formulation of the boundary conditions (9), it can be seen that the boundary $\Gamma_{2}$ is overspecified by prescribing both the temperature $\left.T\right|_{\Gamma_{2}}$ and the flux $\left.\Phi\right|_{\Gamma_{2}}$, whilst the boundary $\Gamma_{1}=\Gamma \backslash \Gamma_{2}$ is underspecified since both the temperature $\left.T\right|_{\Gamma_{1}}$ and the flux $\left.\Phi\right|_{\Gamma_{1}}$ are unknown and have to be determined.

\section{BOUNDARY ELEMENT METHOD}

The Helmholtz equation (1) can also be formulated in integral form, see e.g. Reference [8], as

$$
c(\underline{x}) T(\underline{x})+f_{\Gamma} \frac{\partial E(\underline{x}, \underline{y})}{\partial v(\underline{y})} T(\underline{y}) \mathrm{d} \Gamma(\underline{y})=\int_{\Gamma} E(\underline{x}, \underline{y}) \Phi(\underline{y}) \mathrm{d} \Gamma(\underline{y})
$$

for $\underline{x} \in \bar{\Omega}=\Omega \cup \Gamma$, where the first integral is taken in the sense of the Cauchy principal value, $c(\underline{x})=1$ for $\underline{x} \in \Omega$ and $c(\underline{x})=1 / 2$ for $\underline{x} \in \Gamma$ (smooth), and $E$ is the fundamental solution for the Helmholtz equation (1), which in two dimensions is given by

$$
E(\underline{x}, \underline{y})=\frac{\mathrm{i}}{4} H_{0}^{(1)}(k|\underline{x}-\underline{y}|)
$$

with $H_{0}^{(1)}$ the Hankel function of order zero of the first kind.

A BEM with constant boundary elements, see e.g. References [14,15], is used in order to solve the Cauchy problem for the Helmholtz equation by using the regularization methods described in the next section. If the boundaries $\Gamma_{1}$ and $\Gamma_{2}$ are discretized into $N_{1}$ and $N_{2}$ constant boundary elements, respectively, such that $N=N_{1}+N_{2}$, then on applying the boundary integral equation (10) at each node/collocation point, we arrive at the following system of linear algebraic equations:

$$
\mathbf{A} \underline{T}=\mathbf{B} \underline{\Phi}
$$

Here $\mathbf{A}$ and $\mathbf{B}$ are matrices which depend solely on the geometry of the boundary $\Gamma$ and the vectors $\underline{T}$ and $\underline{\Phi}$ consist of the discretized values of the temperature and the flux on the boundary $\Gamma$. The discretization of the boundary conditions (9) provides the values of $2 \mathrm{~N}_{2}$ of the unknowns and the problem reduces to solving a system of $N$ equations with $2 N_{1}$ unknowns which can be generically written as

$$
\mathbf{C} \underline{X}=\underline{F}
$$

where $\underline{F}$ is computed using the boundary conditions (9), the matrix $\mathbf{C}$ depends solely on the geometry of the boundary $\Gamma$ and the vector $\underline{X}$ contains the unknown values of the temperature and the flux on the boundary $\Gamma_{1}$. 


\section{REGULARIZATION METHODS}

\subsection{Singular value decomposition (SVD)}

In this section, we give a brief description of the SVD and for further details see References $[16,17]$. Consider the ill-conditioned system of linear algebraic equations (12), where $\mathbf{C} \in$ $\mathbb{R}^{N \times 2 N_{1}}, \underline{X} \in \mathbb{R}^{2 N_{1}}, \underline{F} \in \mathbb{R}^{N}$ and assume for the moment that $N \geqslant 2 N_{1}$. Then the SVD of the matrix $\mathbf{C}$ is a decomposition of the form

$$
\mathbf{C}=\mathbf{W} \boldsymbol{\Xi} \mathbf{V}^{\mathrm{T}}=\sum_{i=1}^{2 N_{1}} \underline{w}_{i} \xi_{i} \underline{v}_{i}^{\mathrm{T}}
$$

where $\mathbf{W}=\left(\underline{w}_{1}, \ldots, \underline{w}_{2 N_{1}}\right) \in \mathbb{R}^{N \times 2 N_{1}}$ and $\mathbf{V}=\left(\underline{v}_{1}, \ldots, \underline{v}_{2 N_{1}}\right) \in \mathbb{R}^{2 N_{1} \times 2 N_{1}}$ are matrices with orthonormal columns, i.e. $\mathbf{W}^{\mathrm{T}} \mathbf{W}=\mathbf{V}^{\mathrm{T}} \mathbf{V}=\mathbf{I}_{2 N_{1}}$, and $\boldsymbol{\Xi}=\operatorname{diag}\left(\xi_{1}, \ldots, \xi_{2 N_{1}}\right)$ is a diagonal matrix with non-negative diagonal elements appearing in the order

$$
\xi_{1} \geqslant \xi_{2} \geqslant \cdots \geqslant \xi_{2 N_{1}} \geqslant 0
$$

The numbers $\xi_{i}$ are called the singular values of the matrix $\mathbf{C}$, whilst the vectors $\underline{w}_{i}$ and $\underline{v}_{i}$ are the left and the right singular vectors of the matrix $\mathbf{C}$, respectively. The SVD (14) can be defined for any $N_{1}$ and $N_{2}$ since, if $N_{2}<N_{1}$, we can simply apply the SVD (15) to the matrix $\mathbf{C}^{\mathrm{T}}$.

The most common approach to regularize numerically rank deficient problems is to consider the given matrix $\mathbf{C}$ as a noisy representation of a mathematically rank deficient matrix and to replace $\mathbf{C}$ by a matrix that is close to $\mathbf{C}$ and mathematically rank deficient. The standard choice is the rank- $n$ matrix $\mathbf{C}_{n}$ defined as

$$
\mathbf{C}_{n}=\sum_{i=1}^{n} \underline{w}_{i} \xi_{i} \underline{v}_{i}^{\mathrm{T}}
$$

i.e. we replace the small non-zero singular values $\xi_{n+1}, \ldots, \xi_{2 N_{1}}$ with exact zeros.

The regularized or stabilized TSVD solution $\underline{X}^{(n)}$ is obtained by first replacing the illconditioned matrix $\mathbf{C}$ with the rank- $n$ matrix $\mathbf{C}_{n}$, followed by computing the minimum norm least-squares solution to the problem

$$
\min \|\underline{X}\|_{2} \quad \text { subject to }\left\|\mathbf{C}_{n} \underline{X}-\underline{F}\right\|_{2} \leqslant \varepsilon
$$

where $\varepsilon$ is a measure of the perturbations in the system matrix $\mathbf{C}$ and in the Cauchy data $\tilde{T}$ and $\tilde{\Phi}$ on the boundary $\Gamma_{2}$ and of the incompatibility of the exact solution of the system of equations (12). The optimal truncation number and hence the regularization or stabilization of the numerical solution can be obtained using the discrepancy principle, see Reference [17].

\subsection{Tikhonov regularization method}

In this section, we give a brief description of the Tikhonov regularization method and the choice of the regularization parameter. For further details on this method see References [18-20]. Consider again the ill-conditioned system of linear algebraic equations (12) whose Tikhonov 
regularized solution is given by

$$
\underline{X}_{\lambda}: \mathscr{T}_{\lambda}\left(\underline{X}_{\lambda}\right)=\min _{\underline{X} \in \mathbb{R}^{2 N_{1}}} \mathscr{T}_{\lambda}(\underline{X})
$$

where $\mathscr{T}_{\lambda}$ represents the Tikhonov functional given by

$$
\mathscr{T}_{\lambda}(\underline{X})=\|\mathbf{C} \underline{X}-\underline{F}\|_{2}^{2}+\lambda^{2}\|\mathbf{L} \underline{X}\|_{2}^{2}
$$

and $\mathbf{L} \in \mathbb{R}^{2 N_{1} \times 2 N_{1}}$ induces the smoothing norm $\|\mathbf{L} \underline{X}\|_{2}$ with $\lambda \in \mathbb{R}$ the regularization parameter to be chosen. Formally, the Tikhonov regularized solution $\underline{X}_{\lambda}$ of problem (18) is given as the solution of the regularized equation

$$
\left(\mathbf{C}^{\mathrm{T}} \mathbf{C}+\lambda^{2} \mathbf{L}^{\mathrm{T}} \mathbf{L}\right) \underline{X}=\mathbf{C}^{\mathrm{T}} \underline{F}
$$

Regularization is necessary when solving inverse problems because the simple least-squares solution, i.e. $\lambda=0$, is completely dominated by contributions from data errors and rounding errors. By adding regularization we are able to damp out these contributions and maintain the norm $\|\mathbf{L} \underline{X}\|_{2}$ to be of reasonable size. If too much regularization, or damping, i.e. $\lambda^{2}$ is large, is imposed on the solution then it will not fit the given data $\underline{F}$ properly and the residual norm $\|\mathbf{C} \underline{X}-\underline{F}\|_{2}$ will be too large. If too little regularization is imposed on the solution, i.e. $\lambda^{2}$ is small, then the fit will be good, but the solution will be dominated by the contributions from the data errors, and hence $\|\mathbf{L} \underline{X}\|_{2}$ will be too large.

For the zeroth-order Tikhonov regularization method, i.e. $\mathbf{L}=\mathbf{I}_{2 N_{1}}$, the optimal value of the regularization parameter $\lambda$ and hence the regularization or stabilization of the numerical solution can be obtained using the discrepancy principle, see Reference [17].

\subsection{Conjugate gradient method (CGM)}

In this section, we describe a variational method that can be applied to solve the Cauchy problem for the Helmholtz equation. Since the boundary conditions on the boundary $\Gamma_{1}$ are to be determined, we consider the boundary temperature on the underspecified boundary $\Gamma_{1}$ as a control $v \in H^{1 / 2}\left(\Gamma_{1}\right)$ in a direct problem formulation to fit the Cauchy data $\tilde{T} \in L^{2}\left(\Gamma_{2}\right)$ on the overspecified boundary $\Gamma_{2}$. Therefore, the functional

$$
J: H^{1 / 2}\left(\Gamma_{1}\right) \rightarrow L^{2}\left(\Gamma_{2}\right), \quad v \rightarrow J(v) \equiv \frac{1}{2}\|A v-\tilde{T}\|_{L^{2}\left(\Gamma_{2}\right)}^{2}
$$

is minimized with respect to $v \in H^{1 / 2}\left(\Gamma_{1}\right)$. It has been established that functional (21) is twice Fréchet differentiable and a formula for its first gradient can be obtained by considering an adjoint problem, see Reference [15].

Thus, the CGM applied to our problem has the form of the following algorithm:

Step 1: Set $n=0$ and choose $T^{(0)} \in H^{1 / 2}\left(\Gamma_{1}\right)$.

Step 2: Solve the direct problem

$$
\begin{aligned}
L T\left(T^{(n)}, \tilde{\Phi}\right)(\underline{x}) & =0, & & \underline{x} \in \Omega \\
T\left(T^{(n)}, \tilde{\Phi}\right)(\underline{x}) & =T^{(n)}(\underline{x}), & & \underline{x} \in \Gamma_{1} \\
\left(\partial T\left(T^{(n)}, \tilde{\Phi}\right) / \partial v\right)(\underline{x}) & =\tilde{\Phi}(\underline{x}), & & \underline{x} \in \Gamma_{2}
\end{aligned}
$$

to determine the residual $r^{(n)}=\left.T\left(T^{(n)}, \tilde{\Phi}\right)\right|_{\Gamma_{2}}-\tilde{T}=A T^{(n)}-\tilde{T}$. 
Step 3: Solve the adjoint problem

$$
\begin{aligned}
L \Psi\left(0, r^{(n)}\right)(\underline{x}) & =0, & & \underline{x} \in \Omega \\
\Psi\left(0, r^{(n)}\right)(\underline{x}) & =0, & & \underline{x} \in \Gamma_{1} \\
\left(\partial \Psi\left(0, r^{(n)}\right) / \partial v\right)(\underline{x}) & =r^{(n)}(\underline{x}), & & \underline{x}
\end{aligned}
$$

to determine the gradient $g^{(n)}=-\left.\left(\partial \Psi\left(0, r^{(n)}\right) / \partial v\right)\right|_{\Gamma_{1}}$. Calculate $d^{(n)}$ as follows:

$$
d^{(n)}= \begin{cases}g^{(n)}, & n=0 \\ g^{(n)}+\left(\left\|g^{(n)}\right\|_{H^{1 / 2}\left(\Gamma_{1}\right)}^{2} /\left\|g^{(n-1)}\right\|_{H^{1 / 2}\left(\Gamma_{1}\right)}^{2}\right) d^{(n-1)}, & n \geqslant 1\end{cases}
$$

Step 4: Solve the direct problem

$$
\begin{aligned}
L T\left(d^{(n)}, 0\right)(\underline{x}) & =0, & & \underline{x} \in \Omega \\
T\left(d^{(n)}, 0\right)(\underline{x}) & =d^{(n)}(\underline{x}), & & \underline{x} \in \Gamma_{1} \\
\left(\partial T\left(d^{(n)}, 0\right) / \partial v\right)(\underline{x}) & =0, & & \underline{x} \in \Gamma_{2}
\end{aligned}
$$

to determine $A_{0} d^{(n)}=\left.T\left(d^{(n)}, 0\right)\right|_{\Gamma_{2}}$ and compute

$$
T^{(n+1)}=T^{(n)}+\left(\left\|g^{(n)}\right\|_{H^{1 / 2}\left(\Gamma_{1}\right)}^{2} /\left\|A_{0} d^{(n)}\right\|_{L^{2}\left(\Gamma_{2}\right)}^{2}\right) d^{(n)}
$$

Step 5: Set $n=n+1$. Repeat steps 2-4 until a stopping criterion is prescribed.

As a stopping criterion we choose the one suggested by Nemirovskii [22], namely choose the first $n \in \mathbb{N}$ such that

$$
\left\|r^{(n)}\right\|_{L^{2}\left(\Gamma_{2}\right)} \leqslant \delta \varepsilon
$$

where $\delta>1$ is a constant which can be taken heuristically to be 1.1, as suggested by Hanke and Hansen [22]. It follows from Nemirovskii's result that the above iterative procedure converges with an optimal convergence rate to the exact solution of the problem as the noise level tends to zero.

\subsection{An alternating iterative method}

Apart from general regularization methods which can be applied for solving any ill-posed problem, typical solution methods may be developed for solving particular ill-posed problems. In this section, we describe such a particular regularization algorithm developed for Cauchy problems. This algorithm uses the fact that a part of the boundary, namely $\Gamma_{2}$, is overspecified and the remainder, namely $\Gamma_{1}$, is underspecified in order to reduce the original ill-posed problem to a sequence of well-posed problems by alternating the given data on the overspecified part of the boundary. The iterative algorithm, which was proposed by Kozlov et al. [23] for Cauchy 
problems associated to linear, elliptic, self-adjoint and positive-definite operators, consists of the following steps:

Step 1: Specify an initial approximation $\Phi^{(0)}(\underline{x})$ for the flux on $\Gamma_{1}$ and solve the well-posed mixed boundary value problem

$$
\begin{aligned}
& L T^{(1)}(\underline{x})=0, \quad \underline{x} \in \Omega \\
& \Phi^{(1)}(\underline{x}) \equiv\left(\partial T^{(1)} / \partial v\right)(\underline{x})=\Phi^{(0)}(\underline{x}), \quad \underline{x} \in \Gamma_{1} \\
& T^{(1)}(\underline{x})=\tilde{T}(\underline{x}), \quad \underline{x} \in \Gamma_{2}
\end{aligned}
$$

in order to determine $T^{(1)}(\underline{x})$ for $\underline{x} \in \Omega$ and $T^{(1)}(\underline{x})$ for $\underline{x} \in \Gamma_{1}$.

Step 2: Having constructed the approximation $T^{(2 n-1)}(\underline{x}), n \geqslant 1$, the well-posed mixed boundary value problem

$$
\begin{aligned}
L T^{(2 n)}(\underline{x}) & =0, & & \underline{x} \in \Omega \\
T^{(2 n)}(\underline{x}) & =T^{(2 n-1)}(\underline{x}), & & \underline{x} \in \Gamma_{1} \\
\Phi^{(2 n)}(\underline{x}) & \equiv\left(\partial T^{(2 n)} / \partial v\right)(\underline{x})=\tilde{\Phi}(\underline{x}), & & \underline{x} \in \Gamma_{2}
\end{aligned}
$$

is solved to determine $T^{(2 n)}(\underline{x})$ for $\underline{x} \in \Omega$ and $\Phi^{(2 n)}(\underline{x}) \equiv\left(\partial T^{(2 n)} / \partial v\right)(\underline{x})$ for $\underline{x} \in \Gamma_{1}$.

Step 3: Having constructed the function $T^{(2 n)}(\underline{x}), n \geqslant 1$, the well-posed mixed boundary value problem

$$
\begin{aligned}
L T^{(2 n+1)}(\underline{x}) & =0, & & \underline{x} \in \Omega \\
\Phi^{(2 n+1)}(\underline{x}) & \equiv\left(\partial T^{(2 n+1)} / \partial v\right)(\underline{x})=\Phi^{(2 n)}(\underline{x}), & & x \in \Gamma_{1} \\
T^{(2 n+1)}(\underline{x}) & =\tilde{T}(\underline{x}), & & \underline{x} \in \Gamma_{2}
\end{aligned}
$$

is solved to determine $T^{(2 k+1)}(\underline{x})$ for $\underline{x} \in \Omega$ and $T^{(2 k+1)}(\underline{x})$ for $\underline{x} \in \Gamma_{1}$.

Step 4: Repeat steps 2 and 3 until a prescribed stopping criterion is satisfied.

As a stopping criterion we use again the Morozov discrepancy principle [24] which ceases the iterative procedure described above when the residual norm of the discretized system of equations reaches a threshold value, which is a measure of the perturbations in the matrix of the discretized system of equations and in the Cauchy data, and the incompatibility of the exact solution. A detailed numerical implementation of this algorithm can be found in Reference [14] where it was shown that the iterative algorithm produces a convergent and stable numerical solution for the Cauchy problem considered, provided that a regularizing stopping criterion is used.

\section{NUMERICAL RESULTS AND DISCUSSION}

In this section, we illustrate and compare the numerical results obtained using the regularizing BEMs described in Section 4. In order to present the performance of the numerical methods proposed, we solve the Cauchy problem for a typical benchmark test example in a twodimensional smooth geometry, namely the annular domain $\Omega=\left\{\underline{x}=\left(x_{1}, x_{2}\right) \mid R_{i}^{2}<x_{1}^{2}+x_{2}^{2}<\right.$ $\left.R_{o}^{2}\right\}, R_{i}=0.5$ and $R_{o}=1.0$, since the condition of a smooth domain is required by the 
theoretical analysis of Kozlov et al. [23]. We assume that the boundary $\Gamma$ of the solution domain is divided into two disjointed parts, namely $\Gamma_{1}=\left\{\underline{x} \in \Gamma \mid x_{1}^{2}+x_{2}^{2}=R_{i}^{2}\right\}$ and $\Gamma_{2}=$ $\left\{\underline{x} \in \Gamma \mid x_{1}^{2}+x_{2}^{2}=R_{o}^{2}\right\}$, and consider the following analytical solution for the temperature:

$$
T^{(\mathrm{an})}(\underline{x})=\exp \left(a_{1} x_{1}+a_{2} x_{2}\right), \quad \underline{x}=\left(x_{1}, x_{2}\right) \in \Omega
$$

where $k=\alpha+\mathrm{i} \beta, \alpha=0, \beta=2.0, a_{1}=1.0$ and $a_{2}=\sqrt{\beta^{2}-a_{1}^{2}}$. This example has a flux on the boundary $\Gamma$ given by

$$
\Phi^{(\mathrm{an})}(\underline{x})=\left(a_{1} v_{1}(\underline{x})+a_{2} v_{2}(\underline{x})\right) T^{(\mathrm{an})}(\underline{x}), \quad \underline{x}=\left(x_{1}, x_{2}\right) \in \Gamma
$$

\subsection{Comparison of the numerical results}

It is the purpose of this section to present and compare the numerical results for the Cauchy problem considered in this study which have been obtained using the four regularization methods described in Section 4. In order to investigate the stability and the regularization properties of the numerical methods considered, the boundary data $\left.\tilde{T}\right|_{\Gamma_{2}}$ has been perturbed as

$$
\left.\tilde{T}^{\varepsilon}\right|_{\Gamma_{2}}=\left.\tilde{T}\right|_{\Gamma_{2}}+\delta \tilde{T}, \quad \delta \tilde{T}=\operatorname{G05DDF}(0, \sigma), \quad \sigma=\max _{\Gamma_{2}}|\tilde{T}|(p / 100)
$$

where $\delta \tilde{T}$ is a Gaussian random variable with mean zero and standard deviation $\sigma$, generated by the NAG subroutine G05DDF, and $p$ is the percentage of additive noise included in the input data $\left.\tilde{T}\right|_{\Gamma_{2}}$ in order to simulate the inherent measurements errors. The numerical results presented in this section have been obtained using $N=80$ and $N_{1}=N_{2}=N / 2$ constant boundary elements. These values were found to be sufficiently large such that any further refinement of the mesh size did not significantly improve the accuracy of the results.

Both the CGM and the alternating iterative algorithm described in Section 4.4 require an initial guess to be specified for the temperature or the flux, respectively, on the underspecified boundary $\Gamma_{1}$. This initial guess is improved at every iteration and approaches the exact solution. Therefore, the rate of convergence and the accuracy of these methods clearly depend on how close the initial guess is to the exact solution.

The numerical solutions for the temperature and the flux on the boundary $\Gamma_{1}$ obtained by the CGM for the initial guess $\left.T^{(0)}\right|_{\Gamma_{1}}=0$ and different amounts of noise added into the temperature data $\left.\tilde{T}\right|_{\Gamma_{2}}$ are presented in Figures 1(a) and (b), respectively, in comparison with the exact solution. It should be noted that the example considered in this paper is a very severe example for iterative methods since the exact solution is very far from the most natural guess available. Numerous other examples have been considered and it has been found that the CGM produces very good results for examples for which the initial guess is not too far from the exact solution.

Figures 2(a) and (b) present the numerical solution for the temperature and the flux, respectively, on the boundary $\Gamma_{1}$, retrieved using the alternating iterative algorithm originally proposed by Kozlov et al. [23] with the initial guess $\left.\Phi^{(0)}\right|_{\Gamma_{1}}=0$ in comparison with the exact solution. It can be seen from these figures that, as $p$ decreases, the numerical solution approximates better the exact solution while remaining stable. Even if the numerical results obtained by the alternating iterative algorithm of Kozlov et al. [23] are less accurate than the numerical results obtained by the CGM, they are still a reasonably good approximation to the exact solution of the problem since we have solved a highly ill-posed problem. 

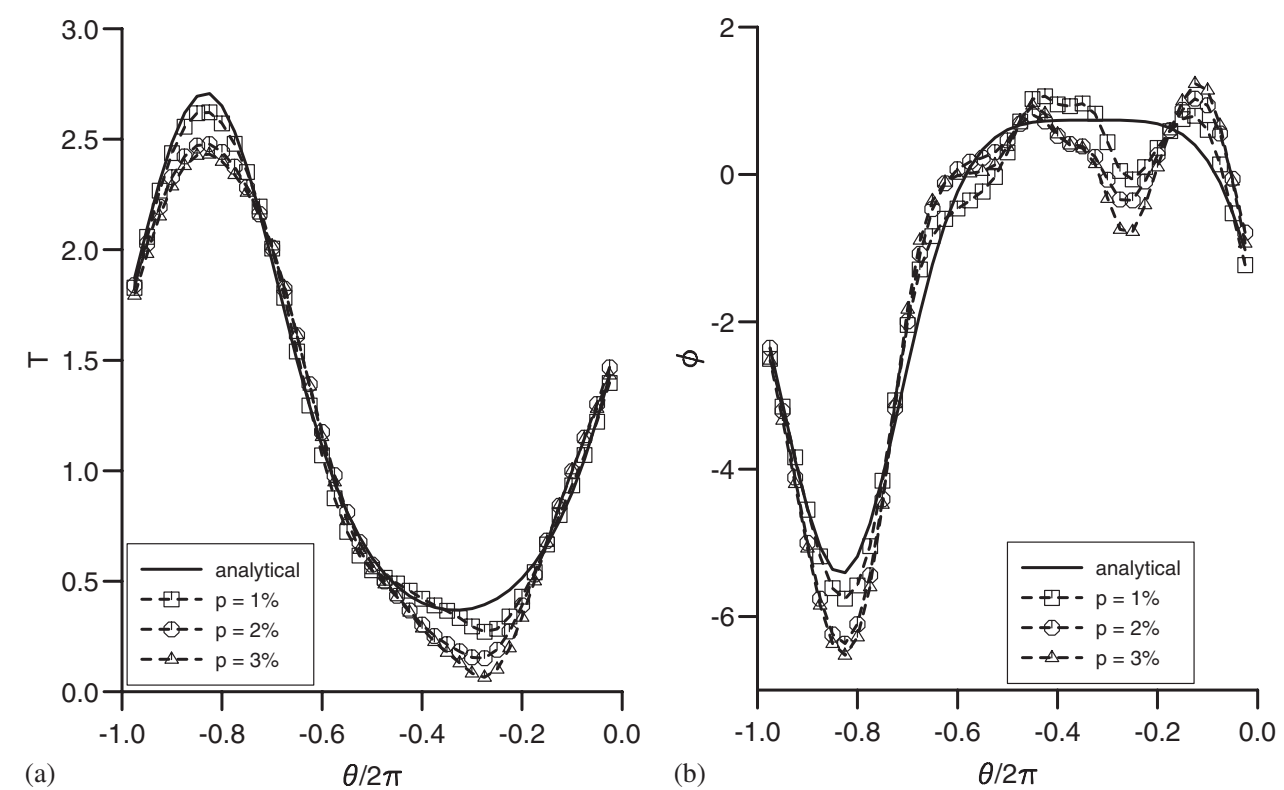

Figure 1. (a) The analytical $T^{(\mathrm{an})}\left(-\right.$ ) and the numerical $T^{(\mathrm{num})}$ temperatures; and (b) the analytical $\Phi^{(\mathrm{an})}\left(-\right.$ and the numerical $\Phi^{(\mathrm{num})}$ fluxes, retrieved on the underspecified boundary $\Gamma_{1}$ by using the CGM for various amounts of noise $p=1 \%(\square), p=2 \%(\bigcirc)$ and $p=3 \%(\triangle)$.

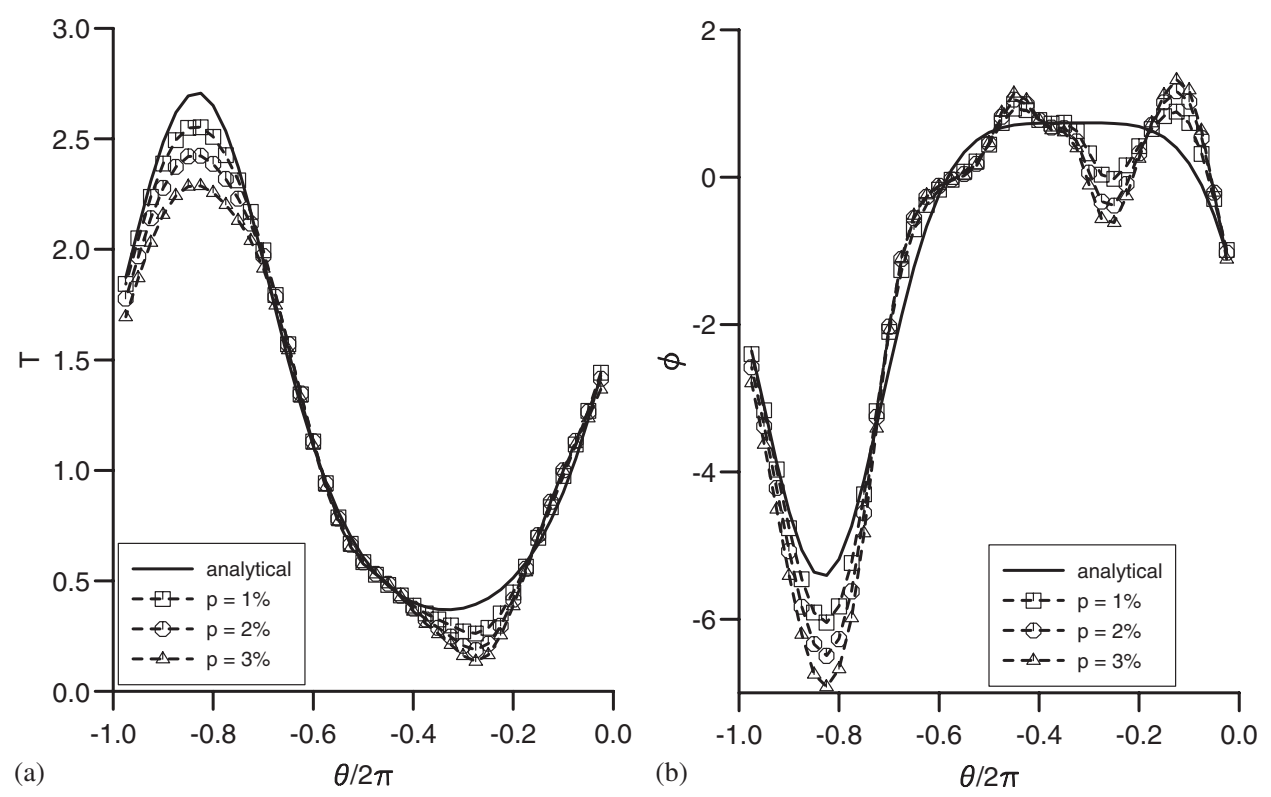

Figure 2. (a) The analytical $T^{(\text {an) }}\left(-\right.$ ) and the numerical $T^{(\mathrm{num})}$ temperatures; and (b) the analytical $\Phi^{(\mathrm{an})}(-)$ and the numerical $\Phi^{(\mathrm{num})}$ fluxes, retrieved on the underspecified boundary $\Gamma_{1}$ by using the alternating iterative algorithm for various amounts of noise $p=1 \%(\square), p=2 \%(\bigcirc)$ and $p=3 \%(\triangle)$. 

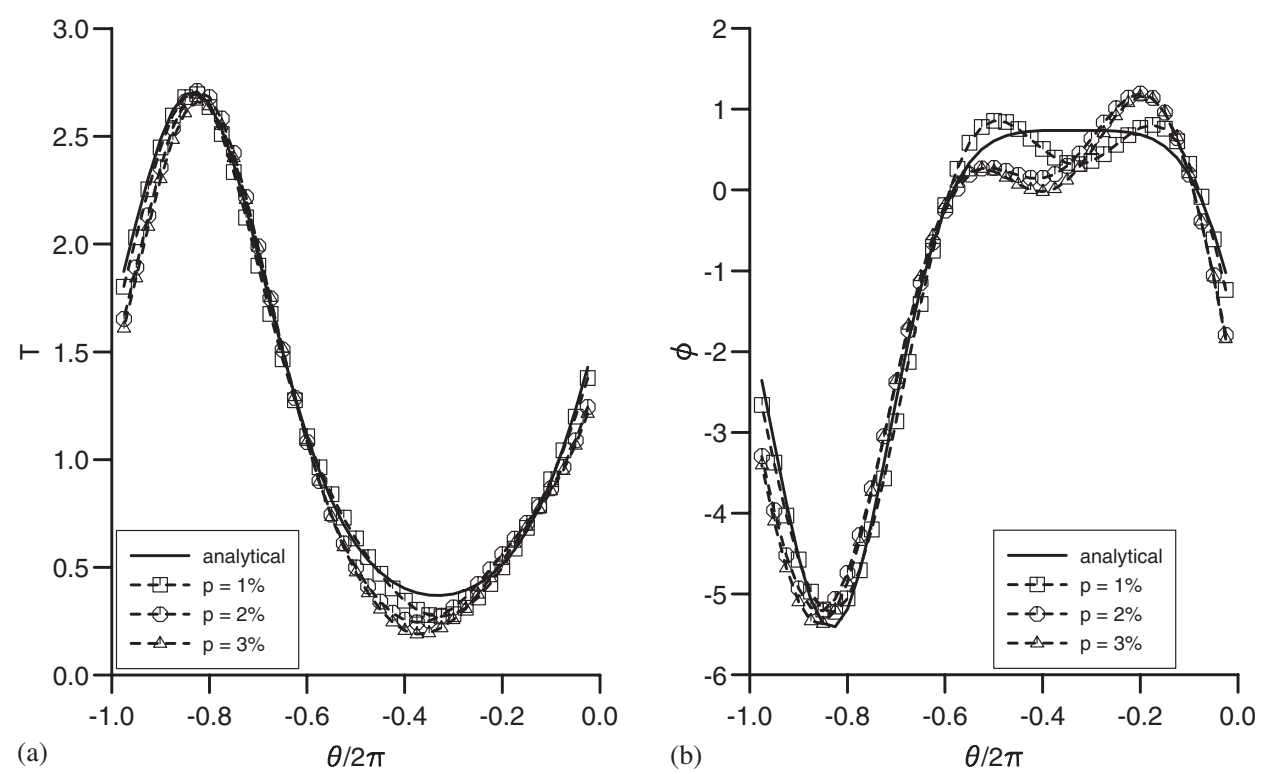

Figure 3. (a) The analytical $T^{(\mathrm{an})}\left(-\right.$ ) and the numerical $T^{(\mathrm{num})}$ temperatures; and (b) the analytical $\Phi^{(\text {an) }}(-)$ and the numerical $\Phi^{(\text {num })}$ fluxes, retrieved on the underspecified boundary $\Gamma_{1}$ by using the SVD method for various amounts of noise $p=1 \%(\square), p=2 \%(\bigcirc)$ and $p=3 \%(\triangle)$.

Although not presented here, it is reported that the Morozov discrepancy principle [24] and the stopping rule proposed by Nemirovskii [21] have been found to be very efficient in choosing the regularization parameter, i.e. the number of iterations at which the numerical methods are stopped, for the alternating iterative method described in Section 4.4 and the CGM, respectively, see Reference $[14,15]$.

The SVD and the Tikhonov regularization methods have been applied to the system of linear algebraic equations (12) in order to simultaneously retrieve the temperature and the flux on the boundary $\Gamma_{1}$. Figures 3(a) and (b) present the numerical solution obtained by using the SVD method for the temperature and the flux, respectively, on the underspecified boundary $\Gamma_{1}$ for various levels of noise, namely $p \in\{1,2,3\}$. It can be seen from these figures that even for large amounts of noise added into the input data there is a very good agreement between the numerical and the exact solution to the problem considered. Figures 4(a) and (b) show the numerical solution for the temperature and the flux, respectively, on the boundary $\Gamma_{1}$ obtained by applying the Tikhonov regularization method to the system of linear algebraic equations (12) for various levels of noise added into the temperature data, namely $p \in\{1,2,3\}$. It should be noted that the numerical solution for the temperature and the flux, respectively, is convergent with respect to decreasing the amount of noise added into the input data and even for large amounts of noise added into the input data, there is a very good agreement between the numerical and the exact solution to the problem considered.

Although not presented here, it is reported that both the SVD and the Tikhonov regularization methods are convergent with respect to the refinement of the mesh size discretization. As a stopping criterion we have used the Morozov discrepancy principle [24] for both the 

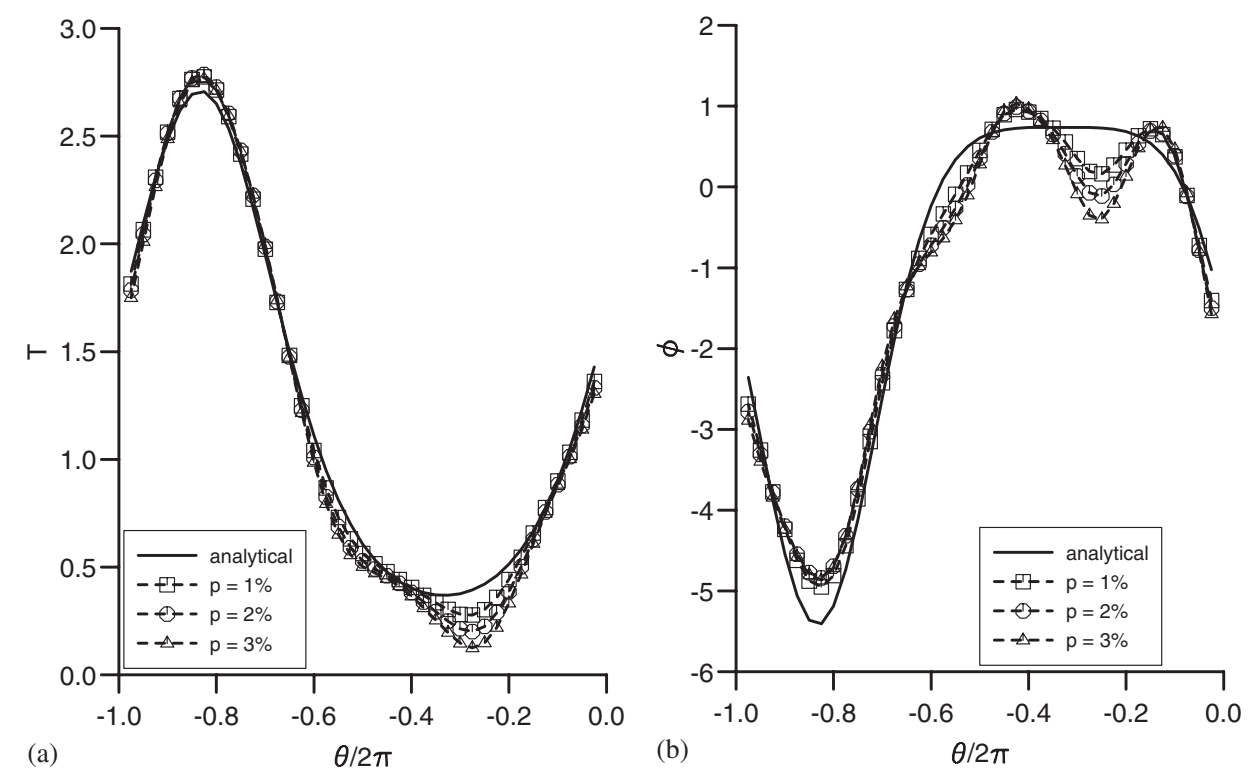

Figure 4. (a) The analytical $T^{(\text {an) }}(-)$ and the numerical $T^{(\text {num })}$ temperatures; and (b) the analytical $\Phi^{(\mathrm{an})}(-)$ and the numerical $\Phi^{(\mathrm{num})}$ fluxes, retrieved on the underspecified boundary $\Gamma_{1}$ by using the Tikhonov regularization method for various amounts of noise $p=1 \%(\square), p=2 \%(\bigcirc)$ and $p=3 \%(\triangle)$.

Tikhonov regularization method and the SVD and this stopping criterion has been found to be very efficient in choosing the regularization parameter and the optimal truncation number, respectively.

In order to compare the four regularization methods considered, Figures 5(a) and (b) present on the same graphs the numerical solution for the temperature and the flux, respectively, on the boundary $\Gamma_{1}$ obtained with each of these methods for $p=2 \%$ noise added into the temperature data $\left.\tilde{T}\right|_{\Gamma_{2}}$. It can be seen from these figures that the most accurate solutions are the ones given by the SVD and the Tikhonov regularization method. Both the CGM and the alternating iterative algorithm of Kozlov et al. [23] give reasonably good approximations for the temperature and the flux on the underspecified boundary $\Gamma_{1}$, but less accurate in comparison with numerical solutions obtained by the SVD and the Tikhonov regularization method. However, for less severe examples and for which we have a better initial guess, it was found that the CGM and the alternating iterative algorithm of Kozlov et al. [23] also produce numerical solutions almost as accurate as the numerical solutions obtained by the SVD and the Tikhonov regularization method. The differences between the regularization methods considered are even larger in the case of the numerical solution for the flux, as can be seen from Figure 5(b).

In Table I we present the accuracy errors

$$
e_{T}=\left\|T^{(\mathrm{num})}-T^{(\mathrm{an})}\right\|_{L^{2}\left(\Gamma_{1}\right)}, \quad e_{\Phi}=\left\|\Phi^{(\mathrm{num})}-\Phi^{(\mathrm{an})}\right\|_{L^{2}\left(\Gamma_{1}\right)}
$$

where $T^{(\mathrm{an})}$ and $\Phi^{(\mathrm{an})}$ are the analytical temperature and flux and $T^{(\mathrm{num})}$ and $\Phi^{(\mathrm{num})}$ are the numerical temperature and flux, respectively, obtained using the regularization methods 

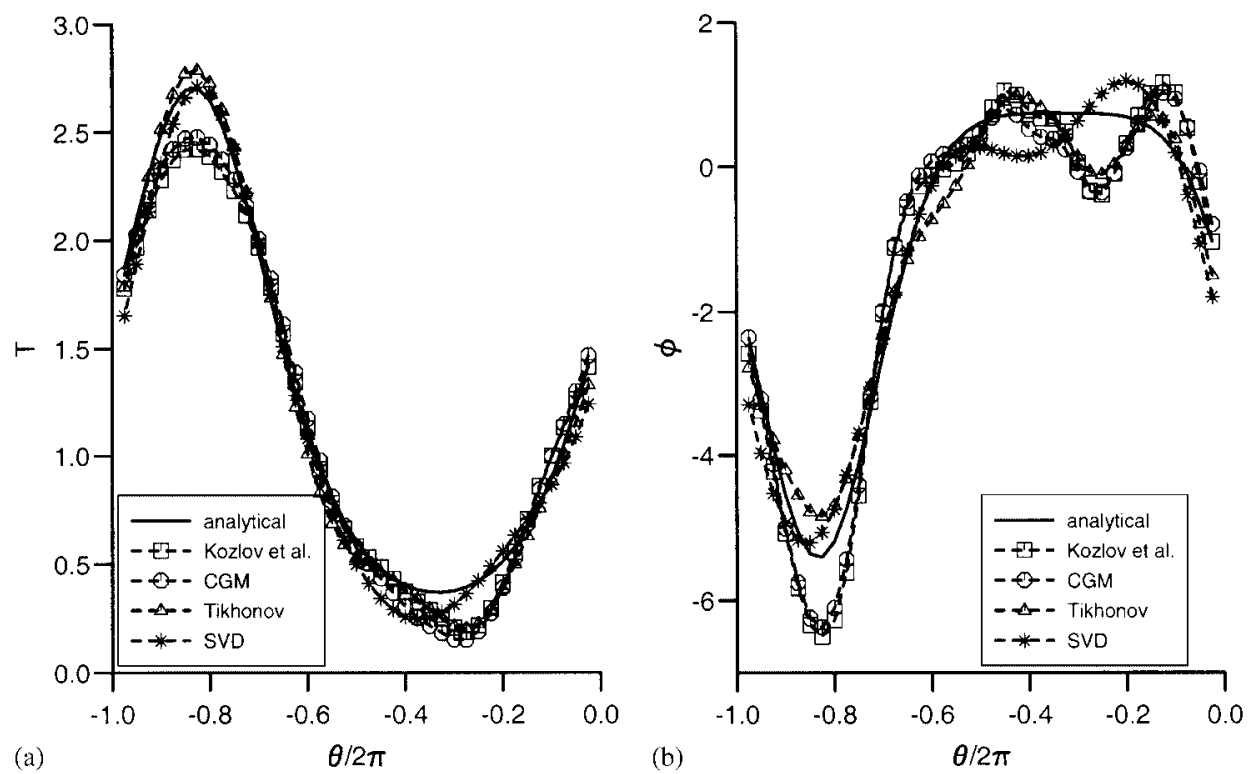

Figure 5. (a) The analytical $T^{\text {(an) }}\left(-\right.$ ) and the numerical $T^{\text {(num) }}$ temperatures; and (b) the analytical $\Phi^{(\text {an })}(-)$ and the numerical $\Phi^{(\text {num })}$ fluxes, retrieved on the underspecified boundary $\Gamma_{1}$ by using the alternating iterative algorithm $(\square)$, the CGM (O), the Tikhonov regularization method $(\triangle)$ and the $\operatorname{SVD}(*)$, for $p=2 \%$ noise.

Table I. The accuracy errors $e_{T}=\left\|T^{(\text {num })}-T^{(\mathrm{an})}\right\|_{L^{2}\left(\Gamma_{1}\right)}$ and $e_{\Phi}=\left\|\Phi^{(\mathrm{num})}-\Phi^{(\mathrm{an})}\right\|_{L^{2}\left(\Gamma_{1}\right)}$ obtained using the four regularization methods described in Section 4 for various levels on noise added into the input data $\left.T^{(\text {an) }}\right|_{\Gamma_{2}}$, namely $p \in\{1,2,3\}$.

\begin{tabular}{lccc}
\hline$p$ & $1 \%$ & $2 \%$ & $3 \%$ \\
\hline$e_{T}($ Kozlov et al. $)$ & $1.30 \times 10^{-1}$ & $2.30 \times 10^{-1}$ & $3.35 \times 10^{-1}$ \\
$e_{T}$ (CGM) & $1.04 \times 10^{-1}$ & $2.24 \times 10^{-1}$ & $2.83 \times 10^{-1}$ \\
$e_{T}$ (Tikhonov) & $1.00 \times 10^{-1}$ & $1.58 \times 10^{-1}$ & $2.09 \times 10^{-1}$ \\
$e_{T}$ (SVD) & $8.24 \times 10^{-2}$ & $1.82 \times 10^{-2}$ & $2.31 \times 10^{-1}$ \\
$e_{\Phi}$ (Kozlov et al. $)$ & $6.51 \times 10^{-1}$ & $1.01 \times 10^{0}$ & $1.31 \times 10^{0}$ \\
$e_{\Phi}$ (CGM) & $6.21 \times 10^{-1}$ & $9.75 \times 10^{-1}$ & $1.22 \times 10^{0}$ \\
$e_{\Phi}$ (Tikhonov) & $5.33 \times 10^{-1}$ & $7.27 \times 10^{-1}$ & $8.86 \times 10^{-1}$ \\
$e_{\Phi}$ (SVD) & $3.77 \times 10^{-1}$ & $7.84 \times 10^{-1}$ & $8.80 \times 10^{-1}$ \\
\hline
\end{tabular}

presented in Section 4 on the underspecified boundary $\Gamma_{1}$ for different levels of noise added into the input data. From this table, it can be seen that the SVD and the Tikhonov regularization method are the regularization methods which provide the most accurate numerical results, whilst the alternating iterative algorithm of Kozlov et al. [23] and the CGM produce less accurate numerical results. 
All the numerical computations have been performed in FORTRAN 90 in double precision on a Pentium $42.4 \mathrm{GHz}$ machine available on the computing system in the School of the Environment at the University of Leeds. It should be noted that the computational times are very good for all the numerical methods analysed in this paper. For example, the lowest computational time, namely $2.46 \mathrm{~s}$, corresponds to the CGM algorithm which performs only three iterations in order to obtain the regularized numerical solution. The SVD and the Tikhonov regularization methods have accounted for almost the same computational time, i.e. 3.37 and $3.49 \mathrm{~s}$, respectively, whilst the alternating iterative algorithm of Kozlov et al. [23] has been found to be the most expensive with respect to the computational cost, namely $10.23 \mathrm{~s}$. However, all the regularizing algorithms require a very low computational effort and hence this represents another advantage of the numerical methods employed to solve the Cauchy problem associated with the Helmholtz equation.

\section{CONCLUSIONS}

In this paper, four regularization methods for the Cauchy problem associated with the Helmholtz equation have been investigated. Three of the methods are general regularization methods, whilst the fourth one is an alternating iterative algorithm developed for Cauchy problems for elliptic linear partial differential equations. It was found that the Cauchy problem for the Helmholtz equation can be regularized by any of the methods considered since all of them produced a stable numerical solution.

However, the numerical solutions obtained by these methods differ in terms of accuracy. It has been found that the CGM outperforms the alternating iterative algorithm which was originally proposed by Kozlov et al. [23] and was presented in Section 4.4. However, these two methods are second best compared to the SVD and the Tikhonov regularization method which produce numerical solutions of almost the same accuracy. We note that for the severe test example considered, the alternating iterative algorithm is less accurate than the other methods considered. The CGM and the alternating iterative algorithm of Kozlov et al. [23] have been found to produce reasonably accurate results for the temperature and, as expected, less accurate numerical results for the flux. However, the SVD and the Tikhonov regularization method have been found to be the methods which produced a good approximation for both the temperature and the flux. Furthermore, all the regularizing algorithms analysed in this paper require a very low computational effort and hence this represents another advantage of the numerical methods employed to solve the Cauchy problem associated with the Helmholtz equation.

\section{ACKNOWLEDGEMENTS}

L. Marin would like to acknowledge the financial support received from the EPSRC.

\section{REFERENCES}

1. Beskos DE. Boundary element method in dynamic analysis: part II (1986-1996). Applied Mechanics Review (ASME) 1997; 50:149-197.

2. Chen JT, Wong FC. Dual formulation of multiple reciprocity method for the acoustic mode of a cavity with a thin partition. Journal of Sound and Vibration 1998; 217:75-95. 
3. Harari I, Barbone PE, Slavutin M, Shalom R. Boundary infinite elements for the Helmholtz equation in exterior domains. International Journal for Numerical Methods in Engineering 1998; 41:1105-1131.

4. Hall WS, Mao XQ. A boundary element investigation of irregular frequencies in electromagnetic scattering. Engineering Analysis in Boundary Elements 1995; 16:245-252.

5. Kern DQ, Kraus AD. Extended Surface Heat Transfer. McGraw-Hill: New York, 1972.

6. Manzoor M, Ingham DB, Heggs PJ. The one-dimensional analysis of fin assembly heat transfer. Journal of Heat Transfer (ASME) 1983; 105:646-651.

7. Wood AS, Tupholme GE, Bhatti MIH, Heggs PJ. Steady-state heat transfer through extended plane surfaces. International Communications in Heat and Mass Transfer 1995; 22:99-109.

8. Chen G, Zhou J. Boundary Element Methods. Academic Press: London, 1992.

9. Bai MR. Application of BEM-based acoustic holography to radiation analysis of sound sources with arbitrarily shaped geometries. Journal of the Acoustical Society of America 1992; 92:533-549.

10. Kim BK, Ih JG. On the reconstruction of the vibro-acoustic field over the surface enclosing an interior space using the boundary element method. Journal of the Acoustical Society of America 1996; 100: 3003-3016.

11. Wang Z, Wu SR. Helmholtz equation-least-squares method for reconstructing the acoustic pressure field. Journal of the Acoustical Society of America 1997; 102:2020-2032.

12. Wu SR, Yu J. Application of BEM-based acoustic holography to radiation analysis of sound sources with arbitrarily shaped geometries. Journal of the Acoustical Society of America 1998; 104:2054-2060.

13. DeLillo T, Isakov V, Valdivia N, Wang L. The detection of the source of acoustical noise in two dimensions. SIAM Journal of Applied Mathematics 2001; 61:2104-2121.

14. Marin L, Elliott L, Heggs PJ, Ingham DB, Lesnic D, Wen X. An alternating iterative algorithm for the Cauchy problem associated to the Helmholtz equation. Computer Methods in Applied Mechanics and Engineering 2003; 192:709-722.

15. Marin L, Elliott L, Heggs PJ, Ingham DB, Lesnic D, Wen X. Conjugate gradient-boundary element solution to the Cauchy problem for Helmholtz-type equations. Computational Mechanics 2003; 31:367-377.

16. Golub GH, van Loan VF. Matrix Computations. The John Hopkins University Press: Baltimore, 1996.

17. Hansen PC. Rank-deficient and Discrete Ill-posed Problems: Numerical Aspects of Linear Inversion. SIAM: Philadelphia, 1998.

18. Tikhonov AN, Arsenin VY. Methods for Solving Ill-posed Problems. Nauka: Moscow, 1986.

19. Hansen PC. Analysis of discrete ill-posed problems by means of the L-curve. SIAM Review 1992; 34:561-580.

20. Hansen PC. The L-curve and its use in the numerical treatment of inverse problems. In Computational Inverse Problems in Electrocardiology, Johnston P (ed.). WIT Press: Southampton, 2001; 119-142.

21. Nemirovskii AS. The regularizing properties of the adjoint gradient method in ill-posed problems. Computational Mathematics and Mathematical Physics 1986; 26:7-16.

22. Hanke M, Hansen PC. Regularization methods for large-scale problems. Surveys Mathematical Industry 1993; 3:253-315.

23. Kozlov VA, Maz'ya VG, Fomin AF. An iterative method for solving the Cauchy problem for elliptic equations. Computational Mathematics and Mathematical Physics 1991; 31:45-52.

24. Morozov VA. On the solution of functional equations by the method of regularization. Soviet Mathematics Doklady 1966; 167:414-417. 